\title{
Investigations of polyethylene glycol mediated ternary molecular inclusion complexes of silymarin with beta cyclodextrins
}

\author{
Mohammad Ansari \\ Department of pharmaceutics, College of pharmacy, Prince Sattam Bin Abdul Aziz University, Al-Kharj, Saudi Arabia.
}

\begin{tabular}{l} 
ARTICLE INFO \\
\hline Article history: \\
Received on: $26 / 07 / 2015$ \\
Revised on: $15 / 08 / 2015$ \\
Accepted on: 09/09/2015 \\
Available online: $27 / 09 / 2015$ \\
\hline Key words: \\
Silymarin, Cyclodextrin, \\
ternary complex, PEG 6000, \\
Solubility, Dissolution rate.
\end{tabular}

\author{
ABSTRACT \\ Objective \\ To evaluate the effect of Polyethylene glycol and $\beta$-cyclodextrin on the solubility and dissolution of silymarin, a \\ poorly water soluble herbal drug. \\ Methodology \\ Effects of beta cyclodextrins and Polyethylene glycol on solubility of silymarin were evaluated by phase \\ solubility studies both separately as well as in combination. Solid inclusion complexes of silymarin $-\beta-$ \\ cyclodextrin in 1:1 molar ratio were prepared with and without polyethylene glycol by kneading method then \\ evaluated for solubility dissolution and physical nature by XRD, FT-IR and NMR spectroscopy studies. \\ Results \\ The aqueous solubility of silymarin was linearly increased as a function of the concentration of $\beta$-cyclodextrin \\ alone and in the presence of polyethylene glycol. Beta cyclodextrin formed molecular inclusion complexes with \\ silymarin in 1:1 molar ratio as revealed by phase solubility diagram. The ternary complexes of silymarin, $\beta$ - \\ cyclodextrin and polyethylene glycol demonstrated better dissolution than those of silymarin and its binary \\ complexes with $\beta$-cyclodextrin alone. Silymarin - $\beta$-cyclodextrin complex caused 1.3 fold increase in the \\ dissolution rate of silymarin, which was further increased up to 1.7 fold in presence of small amount of \\ polyethylene glycol. \\ Conclusion \\ The presence of polyethylene glycol markedly enhances the molecular complexation and solubilizing efficiencies \\ of $\beta$-cyclodextrin on silymarin.
}

\section{INTRODUCTION}

Silymarin (SLM) is mixture of flavonoids and other polyphenolic compounds extracted from the seeds of Silybum marianum (Morazzoni and Bombardelli, 1995). A standardized extract of the seed contains approximately $70-80 \%$ of silymarin flavonolignans and 20-30\% chemically undefined fraction, comprising mostly polymeric and oxidized polyphenolic compounds (Kren and Walterova, 2005). Silymarin is one of the few herbal drugs which have been extensively studied due to its wide pharmacological actions, (Saller et al., 2001; Fraschini et al., 2002; Yang et al., 2003; Rao and Viswanath, 2007). However it exhibits low and variable bioavailability due to its poor aqueous solubility and hence it require enhancement in the

\footnotetext{
* Corresponding Author

Mohammad Ansari, Department of pharmaceutics, College of pharmacy,

Prince Sattam Bin Abdul Aziz University, Al-Kharj, Saudi Arabia.

Email: javedpharma@gmail.com
}

aqueous solubility and dissolution rate to improve its bioavailability and to derive its maximum therapeutic efficacy (Singh and Aggarwal, 2004; Wu et al., 2006). Of the available techniques to enhance solubility of several water insoluble or poorly soluble drugs, use of cyclodextrins to form water soluble inclusion complexes have been proven the most successful due to low cost and their approval by various regulatory agencies (Thomson, 1997; Hedges, 1998). Beta-cyclodextrin (BCD) is the most widely used host molecule for inclusion complexes affecting physical and chemical properties, like solubility and stability of several guest molecules (Fromming and Szejtli, 1994). Unfortunately, the complexation efficiency of BCD is rather low and consequently a significant amount of BCD is required to make inclusion complexes. Moreover, commonly used excipients in drug formulation may further reduce complexation efficiency thus necessitating excessive use of BCD (Loftson et al., 1999), which increases formulation bulk unsuitable for oral administration especially low potency herbal drugs. 
Thus, it is therefore imperative to develop methods that can be applied to enhance the complexation efficiency of BCD. It is reported in a few studies that addition of small amounts of water soluble polymers to cyclodextrin systems have improved both the complexing and solubilizing efficiencies of cyclodextrins (Mura et al., 2001; Koester et al., 2003; Chowdary and Madhavi, 2005). Though cyclodextrins complexation and use of polyethylene glycol (PEG 6000) for enhancing the solubility and dissolution rate of SLM have been investigated individually ( $\mathrm{Li}$ and $\mathrm{Hu}, 2004$; Ghosh et al., 2011), no report is available on their combined use in enhancing the solubility and dissolution rate. In the present investigation the individual main effects and combined (or interaction) effect of BCD and PEG 6000 on the solubility and dissolution rate of SLM were investigated.

\section{MATERIALS AND METHODS}

\section{Chemicals}

Beta cyclodextrin and PEG 6000 were purchased from Sigma Aldrich Chemical Co. USA. Silymarin was purchased from Loba chemie Banglore, India. All other solvents and chemicals were of analytical grade and obtained from Sigma-Aldrich, USA.

\section{Phase solubility studies}

Solubility studies (with BCD, PEG 6000 separately and in combination) were performed according to the method reported by Higuchi and Connors, 1965.

Excess amount of SLM was incubated at $25^{\circ} \mathrm{C}$ and 100 RPM in biological shaker with $0-20 \mathrm{mM}$ of BCD solutions or PEG $6000(0-2 \% \mathrm{w} / \mathrm{v})$ or BCD 0-20 mM containing 0.5\% of PEG 6000.

Suspensions were filtered using 0.45 micron membrane filter after 72 hours and amount of SLM in solutions were analyzed by UV spectrophotometer after appropriate dilution. To nullify the absorbance due to the presence of BCD and PEG 6000, the apparatus were calibrated with the corresponding blank in every measurement.

\section{Preparation of inclusion complexes}

Binary and ternary inclusion complexes were prepared by kneading one mole of Silymarin and beta cyclodextrin with or without PEG $6000(10 \% \mathrm{w} / \mathrm{w})$ in 50:50 mixture of water and ethanol. Mixture was then dried at $60^{\circ} \mathrm{C}$ in hot air oven, pulverized into fine state and passed through sieve \# 80.

\section{Characterization of inclusion complexes}

Prepared inclusion complexes were characterized by using techniques such as X-Ray Differactrometry, FT-IR and NMR spectroscopy.

\section{Powder X-ray diffractometry (X-RD)}

Powder X-ray diffraction pattern of pure drug and inclusion complexes were recorded in X-ray diffractometer (Altima IV, Rigaku, Japan); $\mathrm{Cu}$ radiation, voltage of $40 \mathrm{kV}$ and current of $40 \mathrm{~mA}$. X ray patterns were obtained by scanning from $3^{\circ}$ to $120^{\circ} 2 \theta$ at a step size of $0.02^{\circ}$ with step time of $0.5 \mathrm{~s}$.

\section{Nuclear magnetic resonance spectroscopy}

Pure drug SLM, BCD, binary and ternary inclusion complexes were dissolved in dimethyl sulphoxide. ${ }^{1} \mathrm{H}-\mathrm{NMR}$ spectra were recorded at $300 \mathrm{~K}$ on ultrashield plus $500 \mathrm{MHz}$ NMR spectrometer (Bruker, Massachusetts, USA). Induced changes in the chemical shifts for BCD were calculated in free and bound states.

\section{Fourier transform infra-red spectroscopy}

The Fourier transform infra-red spectroscopy (FT-IR) spectra of pure drug and inclusion complexes were recorded on the FT-IR (Alpha, Germany) using the potassium bromide ( $\mathrm{KBr}$ ) disc technique.

\section{Dissolution studies of inclusion complexes}

In vitro dissolution studies were carried out in USP dissolution apparatus II using $900 \mathrm{ml}$ of the dissolution medium constituting of phosphate buffer $\mathrm{pH} 6.8$ and $1 \%$ sodium lauryl sulphate (SLS) at $37^{\circ} \mathrm{C}$ ). Speed was adjusted to $100 \mathrm{rpm}$. The samples were withdrawn periodically over a period of 2 hours and analyzed using Shimadzu UV spectrophotometer UV-1601.

\section{Results and discussions}

The influence of BCD concentration in the presence or absence of water-soluble polymer PEG 6000 on the solubility of SLM was studied using the method of Higuchi and Connors. This is based on monitoring changes in the solubility of a SLM by the addition of BCD or PEG 6000 or both in combinations.

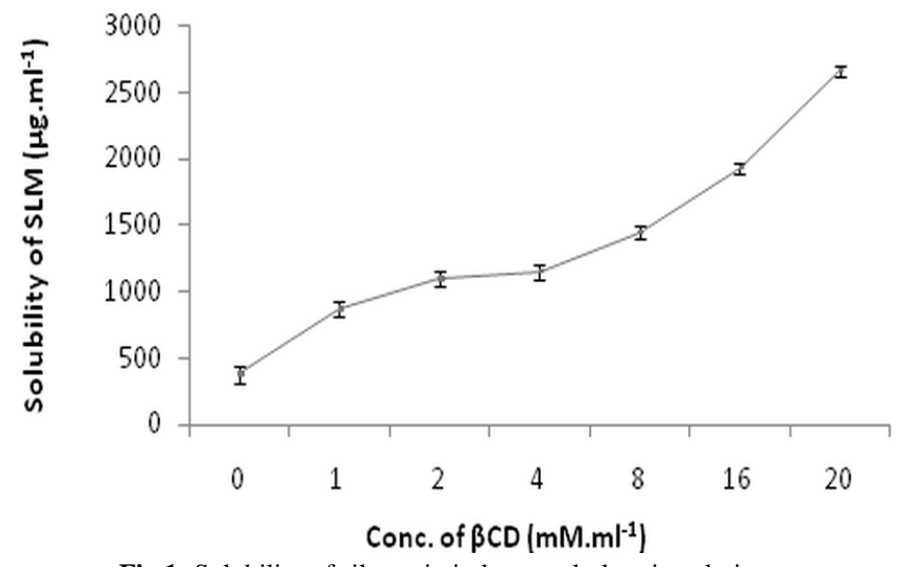

Fig 1: Solubility of silymarin in beta cyclodextrin solutions.

The Phase solubility diagram of SLM in aqueous $\beta C D$ and PEG 6000 solutions were shown in Fig.1 and Fig 2 respectively. Both demonstrated AL type equilibrium phase solubility diagram as SLM solubility increases linearly as a function of BCD and PEG 6000 concentrations.

Ternary phase-solubility study diagram obtained in presence PEG 6000 also displayed $\mathrm{A}_{\mathrm{L}}$ type (Higuchi and Connors, 
1965) equilibrium phase solubility diagrams (Fig.3), indicating that SLM solubility increase linearly as a function of BCD concentration in the presence of PEG and that soluble complexes were formed without occurrence of precipitation in the range of BCD concentration used.

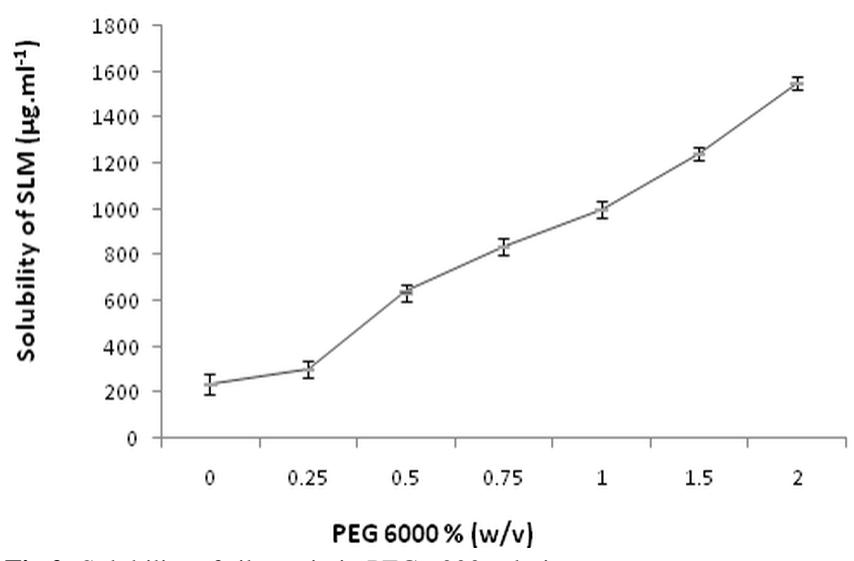

Fig 2: Solubility of silymarin in PEG 6000 solutions.

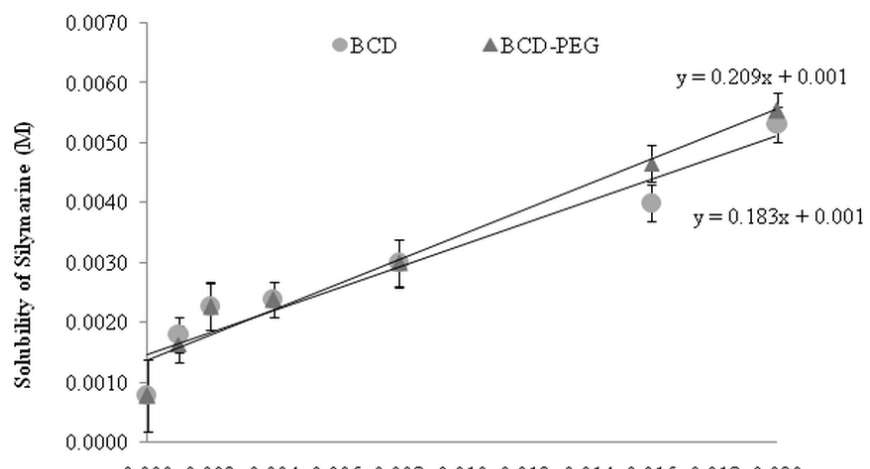

$\begin{array}{llllllllllllllll}0.000 & 0.002 & 0.004 & 0.006 & 0.008 & 0.010 & 0.012 & 0.014 & 0.016 & 0.018 & 0.020\end{array}$ Concentration of BCD $(\mathrm{M}$

Fig 3: Phase solubility diagram of silymarin in binary and ternary mixtures.

The slope values were found to be less than one suggesting the formation of 1:1 stoichiometry complexes. The apparent stability constant $(\mathrm{Kc})$ in each case was calculated from the slope of the corresponding linear plot of the phase solubility diagram according to the equation, $\mathrm{Kc}=$ Slope/So (1-Slope), where So is the solubility of the drug in the absence of solubilizers. Complexation efficiency (CE) is considered more accurate method for determination of the solubilizing effect of cyclodextrins as it is independent of both So and the intercept. It is more reliable when the influences of various pharmaceutical excipients such as polymers on the solubilization of drug are to be investigated. Complexation efficiency is the concentration ratio between cyclodextrin in a complex and free cyclodextrin. It is calculated from the slope of the phase-solubility diagrams by using following equations:

$$
\begin{gathered}
\mathrm{Kc}=\text { Slope } / \text { So }(1-\text { Slope })-1 \\
\mathrm{CE}=\text { Slope } /(1 \text { - Slope })-2
\end{gathered}
$$

The estimated apparent stability constants and complexation efficiency values of complexes are given in Table 1.
Table 1: Effect of PEG 6000 on solubilizing and complexation efficiency of beta cyclodextrin over silymarin.

\begin{tabular}{lll}
\hline PARAMETERS & BCD & BCD + PEG 6000 \\
\hline Slope* $*$ & 0.183 & 0.209 \\
Stability constant $(\mathrm{Kc}) \dagger$ & 286.074 & 337.812 \\
Complexation efficiency (CE) $\dagger$ & 0.224 & 0.264 \\
Solubilizing efficiency (SE) I] & 6.789 & 7.095 \\
\hline
\end{tabular}

*Slope taken from linear equation of phase solubility diagrams; $\dagger \mathrm{Kc}$ : Calculated as $\mathrm{Kc}=$ Slope/So (1-Slope), $\ddagger$ CE: calculated as Slope $/(1-$ Slope $)$ of respective phase solubility diagrams; II SE: Ratio between drug solubility in aqueous solution with or without solublizers in water.

It has been observed that addition of PEG to the BCD solution did not change the type of phase-solubility diagrams obtained for binary systems and resulted in increase in stability constant. The observed enhancement of stability constant with addition of the PEG shows that the PEG is able to interact with SLM-BCD binary complexes. The solubilizing effect of BCD was increased in the presence of PEG; consequently, a synergistic effect in SLM solubility was observed in the presence of BCD and PEG.

\section{$X$ ray diffraction study}

$\mathrm{X}$ ray diffraction study represents a first choice analytical tool for an accurate physicochemical characterization of drug- $\beta$-CD systems in the solid state and is commonly used as a routine method for a rapid preliminary qualitative investigation of the crystal behaviour of the single components, their physical mixtures and the inclusion compound candidate prepared according to a variety of standard procedures.

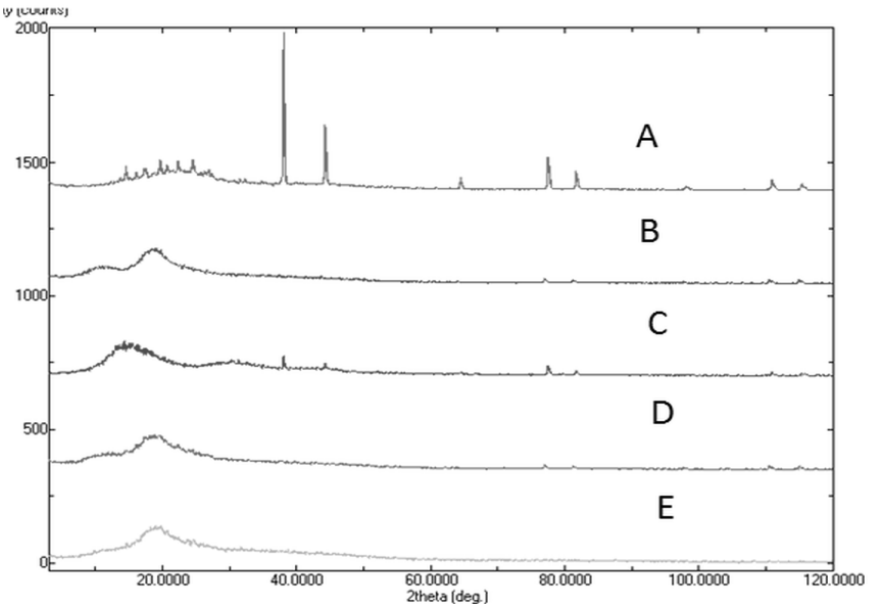

Fig. 4:X-ray diffractograms of silymarin and inclusion complexes. ASilymarin, B- beta cyclodextrin, C- PEG 6000, D- silymarin: beta cyclodextrin binary complexes, E- silymarin: beta cyclodextrin: PEG 6000 ternary complexes.

The purpose is to provide evidence that differences between the physical mixtures and the putative inclusion complex exist. X ray diffractrograms of pure SLM, physical mixtures (PM), binary and ternary inclusion complex prepared by kneading method (KD) are shown in Fig.4. These curves were indicative of 
formation of inclusion complex in solid state. The disappearance of sharp peaks of SLM might be attributed to an amorphous state and/or to an inclusion complexation.

\section{Nuclear magnetic resonance spectroscopy}

The interaction between SLM and beta-cyclodextrin were studied by $1 \mathrm{H}$ NMR spectra. The significant distinction between the $1 \mathrm{H}$ NMR spectra of SLM and the inclusion complex of betacyclodextrin with SLM in DMSO as depicted in Fig.5, indicated that the inclusion complex was formed. The chemical shifts values of for different protons in BCD and SLM: BCD inclusion complex are shown in Table 2.

Table 2: Chemical shifts of protons of beta cyclodextrin in free and bound state.

\begin{tabular}{|c|c|c|c|c|c|}
\hline \multirow[b]{2}{*}{$\begin{array}{l}\text { Z } \\
0 \\
0 \\
0 \\
0 \\
0\end{array}$} & \multicolumn{5}{|c|}{ Chemical shift of BCD in pure and complexed states $(\delta)$} \\
\hline & $\underset{0}{0}$ & 氞芦 & ${ }^{\infty}$ & 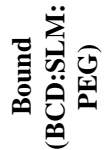 & 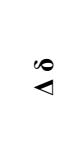 \\
\hline $\mathrm{H}-1$ & 5.734 & 5.759 & 0.025 & 5.755 & 0.021 \\
\hline $\mathrm{H}-2$ & 3.342 & 3.359 & 0.017 & 3.358 & 0.016 \\
\hline $\mathrm{H}-3$ & 5.692 & 5.709 & 0.017 & 5.705 & 0.013 \\
\hline $\mathrm{H}-4$ & 3.324 & 3.339 & 0.015 & 3.337 & 0.013 \\
\hline $\mathrm{H}-5$ & 4.516 & 4.522 & 0.006 & 4.513 & -0.003 \\
\hline H-6 & 4.824 & 4.841 & 0.017 & 4.839 & 0.015 \\
\hline
\end{tabular}

Significant changes in microenvironment of free and bound states of drugs causes changes in chemical shift of host or guest nucleus which in turn could provide evidence for the formation of the inclusion complexes (Fernandes et al 2003). The 1H NMR shifts are in agreement with those reported in the literatures, which mentioned that downfield shifts should be observed for guest protons and upfield shifts for host protons upon hydrophobic interactions between both partners (Thakkar and Demarco 1971; Fernandes et al 2003).

\section{Fourier transform infra-red spectroscopy}

FT-IR curves of pure components; physical mixtures, binary and ternary inclusion complex prepared by kneading method are shown in Fig.6. These curves were indicative of formation of inclusion complex in solid state. The disappearance of sharp peaks of SLM is attributed to an amorphous state and/or to formation of inclusion complexes.

\section{In vitro dissolution profiles}

The dissolution rate of SLM alone and from its inclusion complexes was carried out in USP dissolution apparatus II. Dissolution profiles of all tested samples are shown in Fig. 7. The dissolution of silymarin was rapid and higher from both binary and ternary cyclodextrin inclusion complexes prepared when compared to pure drug. The dissolution studies revealed that about $55 \%$ and $73 \%$ SLM were dissolved from pure silymarin and binary inclusion complexes whereas ternary inclusion complex exhibited more than $93 \%$ release in the same time period. Water-soluble polymers increase the solubilizing effect of cyclodextrins by increasing Kc of the SLM-BCD complexes, as reported by several authors (Loftsson 1998; Savolainen et al., 1998). This increase enhances the complexation efficiency and thus less cyclodextrin is needed to solubilize a given amount of drug when PEG 6000 was present in the aqueous media. In the present work addition of small amount of PEG 6000 to cyclodextrin solutions resulted in higher Ks values for ternary complexes in comparison to binary complexes suggesting a significant improvement in the complexation efficiency between SLM-BCD thereby markedly enhancing the solubilizing efficiency of BCD and this is in agreement with previous reports (Savolainen et al., 1998; Cappello et al., 2001). In vitro studies in distilled water for inclusion complexes of BCD with PEG 6000 showed increase in rates of dissolution several times higher than those of SLM and its complexes with BCD alone. These finding confirms that the addition of small amounts of PEG 6000 improves solubilizing and complexing ability of cyclodextrin which further related to increased release of drug in dissolution medium. Study signifies the use of PEG 6000 in combination with BCD for the formation of inclusion complex of SLM.

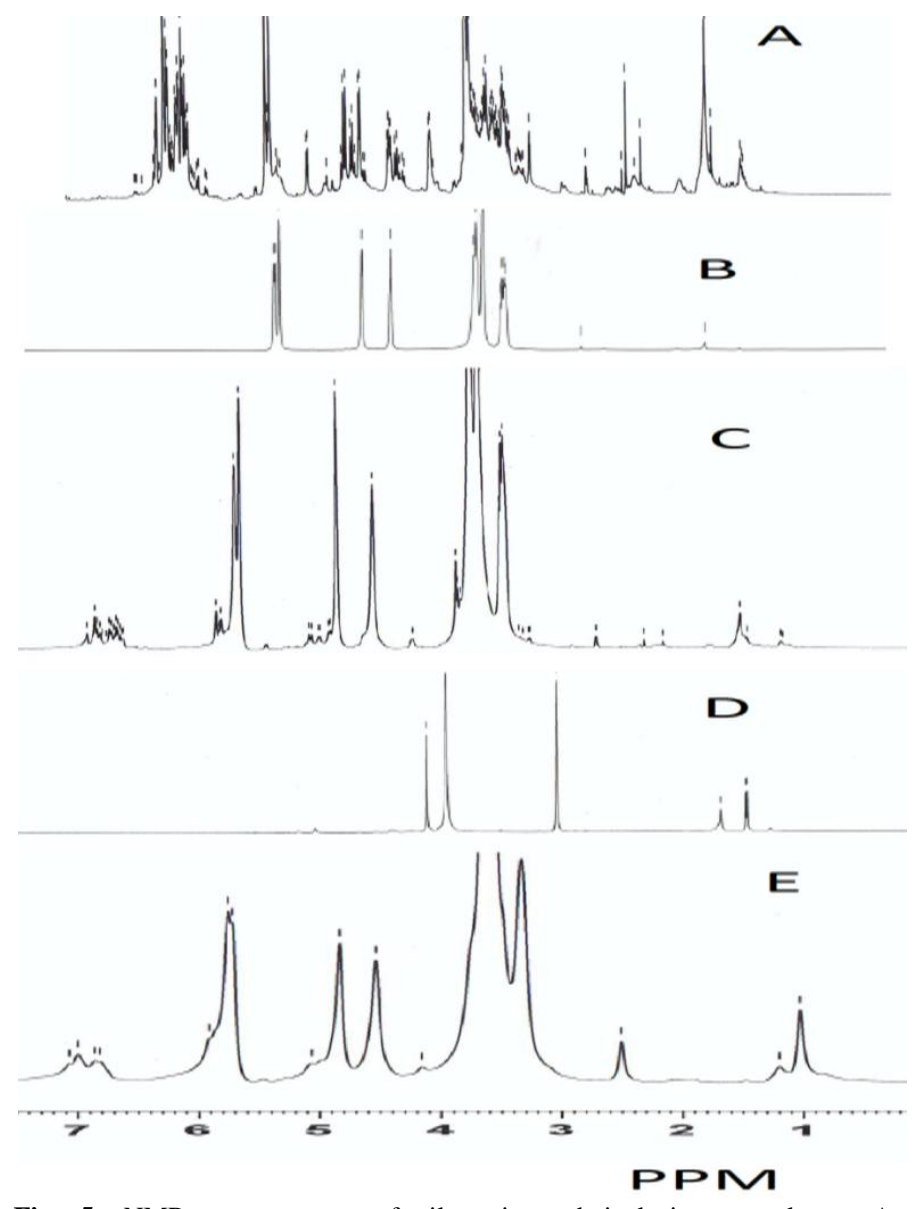

Fig. 5: NMR spectrograms of silymarin and inclusion complexes. ASilymarin, B- beta cyclodextrin, C- silymarin: beta cyclodextrin binary inclusion complexes, D- PEG 6000, E- silymarin: beta cyclodextrin: PEG 6000 ternary inclusion complexes. 

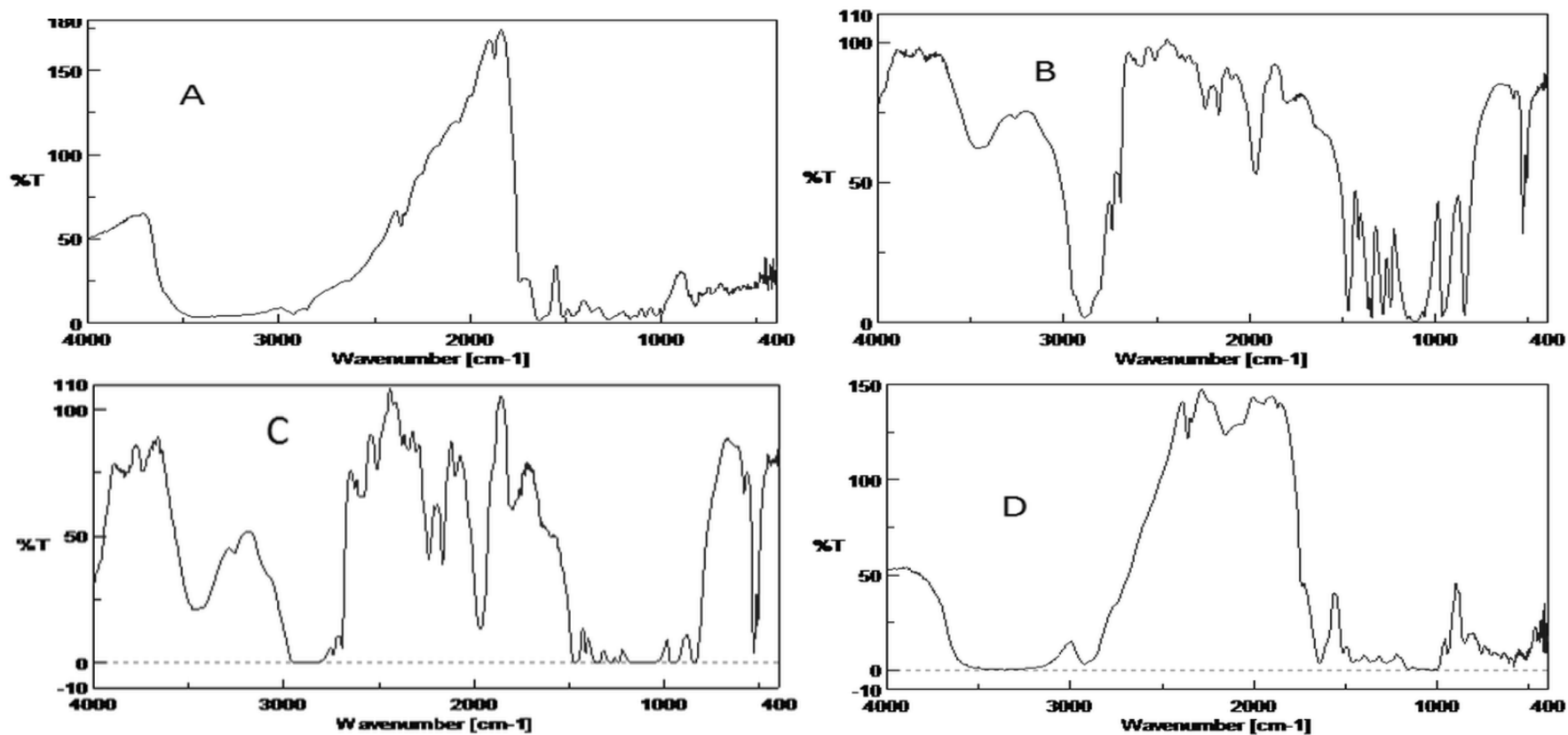

Fig.6: FT-IR spectrograms of silymarin and inclusion complexes. A- Silymarin, B- beta cyclodextrin, C- PEG 6000, D- silymarin: beta cyclodextrin: PEG 6000 ternary inclusion complexes.

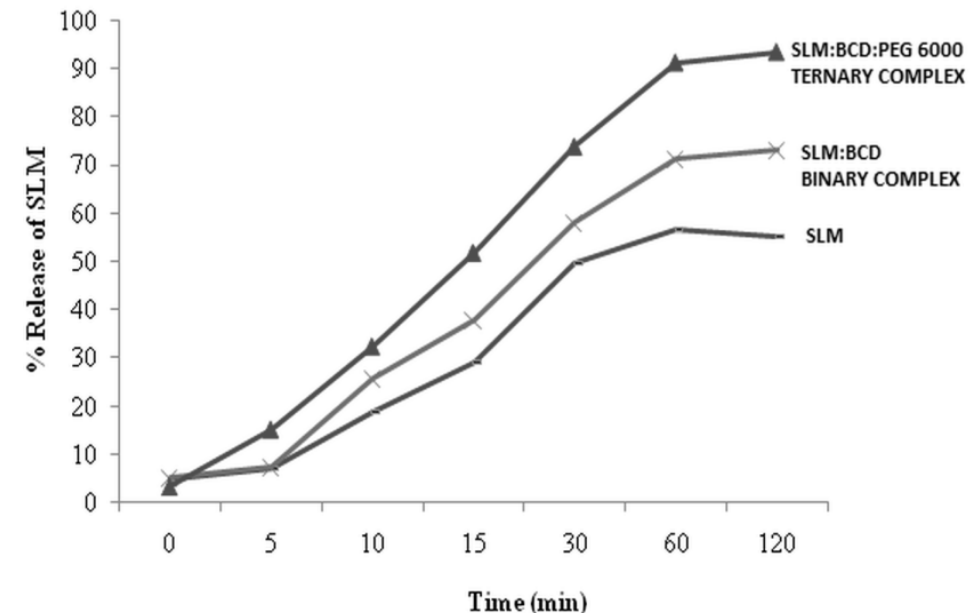

Fig.7: In vitro Release profiles of silymarin and inclusion complexes. (一) silymarin, (X) silymarin: beta cyclodextrin binary inclusion complexes, ( $\mathbf{\Delta})$ silymarin: beta cyclodextrin: PEG 6000 ternary inclusion complexes.

\section{CONCLUSION}

The complexation of SLM with BCD in absence or presence of PEG 6000 was investigated by phase solubility studies. The aqueous solubility of SLM was increased linearly as a function of the concentration of BCD and PEG 6000. The values of stability constant indicated that all the complexes formed between SLM and BCD was quite stable. The values of stability constant of BCD were found to be higher in the presence of PEG 6000 indicating higher complexation and solubility efficiencies.

\section{ACKNOWLEDGEMENT}

Author is grateful to the Dean, College of Pharmacy, for providing opportunity, resources, instrumentation and facility to carryout and complete this research project and also to the colleagues for their help and support.

\section{Declaration /Conflict of interest policy}

Author declares that there is no conflict of interest.

\section{REFERENCES}

Morazzoni P, Bombardelli E.. Silybum marianum (Carduus marianus). Fitoterapia, 1995; 64: 3-42.

Kren V, Walterova D. Silybin and Silymarin - New effects and applications. Biomed Papers, 2005; 149: 29-41.

Saller R, Meier R, Brignoli R. Use of silymarin in the treatment of liver diseases. Drugs, 2001; 61: 2035-2063.

Fraschini F, Demartini G, Esposti D. Pharmacology of silymarin. Clin Drug Investig, 2002; 22: 51-65. 
Yang SH, Lin JK, Chen WS, Chiu JH. Antiangiogenic effect of silymarin on colon cancer LoVo cell line. J Surg Res, 2003; 113:133-138.

Rao PR, Viswanath RK. Cardioprotective activity of silymarin in ischemia-reperfusion-induced myocardial infarction in albino rats. Exp Clin Cardiol, 2007; 12: 179-187.

Wu W, Wang Y, Que L. Enhanced bioavailability of silymarin by self-microemulsifying drug delivery system. Eur J Pharm Biopharm, 2006; 63: 288-294.

Singh RP, Aggarwal R. Prostate cancer prevention by silibinin. Curr Cancer Drug Targets, 2004; 4:1-11.

Thomson Do. Cyclodextrins--enabling excipients: their present and future use in pharmaceuticals. Crit Rev Ther Drug Carries Syst, 1997; 14: 1-104.

Hedges AR. Industrial Applications of Cyclodextrins. Chem Rev, 1998; 98: 2035-2044.

Fromming KH, Szejtli J. 1994. Cyclodextrins Inclusion Complexes. In: Fromming KH, Szejtli J, eds. Cyclodextrin in Pharmacy. Dordrecht: Kluwer Academic Publishers 45-81.

Loftsson T, Masson M, Sigurjonsdottir JF. Methods to enhance the complexation efficiency of cyclodextrins. S T P Pharma Sci, 1999; 9: 237-242.

Mura P, Faucci MT and Bettinetti GP. The influence of polyvinylpyrrolidoneon naproxen complexation with hydroxypropyl-betacyclodextrin. Eur J Pharm Sci, 2001; 13: 187-194.

Koester LS, Mayorga P, Bassani VL.. Carbamzepine/betaCD/ HPMC solid dispersions. I. Influence of the spray-drying process and betaCD/HPMC on the drug dissolution profile. Drug Dev Ind Pharm, 2003; 29: 139-144.

Chowdary KPR, Madhavi BLR. Novel drug delivery technologies for insoluble drugs. Indian Drugs, 2005; 42: 557-563.

Ghosh A, Biswas S, Ghosh T. Preparation and evaluation of silymarin $\beta$-cyclodextrin molecular inclusion complexes. J Young Pharm, 2011; 3: 205-210.
$\mathrm{Li} \mathrm{FQ}$, $\mathrm{Hu} \mathrm{JH}$. Improvement of the dissolution rate of silymarin by means of solid dispersions. Chem Pharm Bull, 2004; 52: 972 983.

Higuchi T, Connors KA. 1965. Phase-Solubility Techniques. In: Reill CN, ed. Advances in Analytical Chemistry and Instrumentation. New York: Wiley-Inter sciences, 117-212.

Fernandes CM, Carvalho RA, Pereira da Costa S, Veiga FJ. Multimodal molecular encapsulation of nicardipine hydrochloride by $\beta$ cyclodextrin, hydroxypropyl- $\beta$-cyclodextrin and triacetyl- $\beta$-cyclodextrin in solution structural studies by $1 \mathrm{H}$ NMR and ROESY experiments. Eur J Pharm Sci, 2003; 18: 285-296.

Thakkar AL, Demarco PV. Cycloheptaamylose inclusion complexes of barbiturates: correlation between proton magnetic resonance and solubility studies. J Pharm Sci, 1971; 60: 652-653.

Loftsson T. Increasing the cyclodextrin complexation of drugs and drug bioavailability through addition of water-soluble polymers. Pharmazie, 1998; 53: 733-740.

Savolainen J, Jarvinen K, Taipale H, Jarho P, Loftsson T, Jarvinen T. Co-administration of a water-soluble polymer increases the usefulness of cyclodextrins in solid oral dosage forms. Pharma Res, 1998; 15: 1696-1701.

Cappello B, Carmignani C, Iervolino M, La Rotonda MI, Saettone MF. Solubilization of tropicamide by hydroxypropyl-betacyclodextrin and water-soluble polymers: in vitro/in vivo studies. Int $\mathrm{J}$ Pharm, 2001; 213: 75-81.

\section{How to cite this article:}

Mohammad Ansari. Investigations of polyethylene glycol mediated ternary molecular inclusion complexes of silymarin with beta cyclodextrins. J App Pharm Sci, 2015; 5 (09): 026-031. 